\title{
DETERMINAÇÃO DO PERFIL BIOQUÍMICO DE RATOS SUBMETIDOS A ACUPUNTURA E LASER ACUPUNTURA NOS PONTOS ZUSANLI E YINTANG
}

\author{
Iara Alves Coelho ${ }^{1}$ \\ Caroline Valente ${ }^{2}$ \\ Murilo Luiz Cerutti ${ }^{3}$
}

\begin{abstract}
Resumo: Terapias complementares, como acupuntura e laser acupuntura, têm se destacado na prática médica devido as recomendações da Organização Mundial da Saúde (OMS), uma vez que essas terapias apresentam efeitos orgânicos positivos. Objetivo comparar acupuntura e laser acupuntura nos acupontos E36 (Zusanli) e EX3 (Yintang) no perfil lipídico e glicêmico em ratos em jejum e sem jejum, bem como nos níveis séricos de cortisona. Ratos Wistar foram divididos em dois grupos (com jejum e sem jejum) e mantidos em biotério com ciclo claro-escuro (12-12 horas), temperatura controlada $\left(22 \pm 2^{\circ} \mathrm{C}\right)$ e livre acesso à água e comida. Os animais permaneceram no laboratório de bioquímica por um período de $1 \mathrm{~h}$ para aclimatação. Verificou-se que a laser acupuntura no grupo sem jejum houve aumento significativo nos níveis de colesterol total (CT, 32\%) em relação ao controle e acupuntura, HDL-colesterol (HDL-c, 78\%) e glicose (18\%) apenas ao controle; no grupo com jejum a terapia com laser acupuntura foi observado um aumento significativo no CT e no HDL-c (38 e $39 \%$, respectivamente) e uma diminuição significativa nos níveis séricos de cortisol (30\%), todos em relação ao controle. O presente estudo demonstrou respostas significativas com o tratamento a laser nos acupontos E36 (Zusanli) e EX3 (Yintang), quando comparados aos grupos acupuntura e controle. Os presentes achados reforçam a hipótese de que as terapias complementares têm efeitos significativos que podem beneficiar o paciente e devem, portanto, ser estimuladas sempre que possível.
\end{abstract}

Palavras chave: acupuntura, laser acupuntura, perfil lipídico, perfil glicêmico, cortisol

\section{DETERMINATION OF THE BIOCHEMICAL PROFILE OF RATS SUBMITTED TO ACUPUNCTURE AND LASER ACUPUNCTURE IN POINTS ZUSANLI AND YINTANG}

\begin{abstract}
Complementary therapies, such as acupuncture and Acupuncture Laser, have been stands out in medical practice due to World Health Organization (WHO) advice, since these therapies presents positive organic effects. Objective compare acupuncture and laser acupuncture effect at ST36 (Zusanli) and EX3 (Yintang) acupoints on lipidic and glycemic profile in fasting and fed rats, as well as, in the serum cortisol levels. Wistar rats were divided into two groups (fasted and fed) and were maintained in a vivarium with lightdark cycle (12-12 hours), controlled temperature $\left(22 \pm 2^{\circ} \mathrm{C}\right)$ and free access to water and food. Animals remained in the biochemistry laboratory for a period of $1 \mathrm{~h}$ for acclimatization. It was verified that acupuncture laser in the group without fasting had a significant increase in the levels of total cholesterol (TC, 32\%) in relation to control and acupuncture, HDL-cholesterol (HDL-c, 78\%) and glucose (18\%) to control; in the group with fasting acupuncture laser therapy a significant increase was observed in TC and HDL-c (38 and 39\%, respectively) and a significant decrease in serum cortisol levels (30\%), all in relation to the control. The present study demonstrated significant responses with laser treatment in the ST36 (Zusanli) and EX3 (Yintang) acupoints, when compared to acupuncture and control group. The present findings reinforce the hypothesis that complementary therapies have significant effects that may benefit the patient and should, therefore, be stimulated whenever possible.
\end{abstract}

Keywords: acupuncture, laser acupuncture, lipid profile, glycemic profile, cortisol

\footnotetext{
${ }^{1}$ Professora no Departamento de Medicina da Universidade Regional de Blumenau (FURB), Blumenau-SC, Brasil.

2 Professora no Departamento de Ciências Naturais da Universidade Regional de Blumenau (FURB), Blumenau-SC, Brasil.

${ }^{3}$ Professora no Departamento de Medicina da Universidade Regional de Blumenau (FURB), Blumenau-SC, Brasil.
} 


\section{Introdução}

No ano de 2006, o Ministério da Saúde criou a Política Nacional de Práticas Integrativas e Complementares (PNPIC) no Sistema Único de Saúde (SUS) com o intuito de estimular a diminuição do consumo de medicamentos e substituir, lenta e gradativamente os modelos de atenção voltados à doença e à intervenção medicamentosa, por estratégias que garantam a integralidade na atenção à saúde e o envolvimento de intervenções profiláticas, promoção em saúde e qualidade de vida (BRASIL, 2006).

Diante disso, as terapias complementares passam a ter um destaque importante e de acordo com a Organização Mundial de Saúde (OMS), a acupuntura que é uma técnica da Medicina Tradicional Chinesa (MTC), vem sendo utilizada para o tratamento de desequilíbrios energéticos, funcionais e até mesmo orgânicos. O objetivo dessa terapia é favorecer, através de estímulos produzidos por agulhas, a criação de condições internas para o retorno do equilíbrio do organismo como um todo, alívio de suas desordens, sem apenas o emprego da farmacologia típica (COSTA, 2017).

Os pontos de acupuntura são meios de comunicação entre o exterior e o interior do corpo, estando sujeito às influências das emoções e clima que o indivíduo está exposto. Esses pontos comunicam-se com os canais de energia e fazem circular Qi (energia) e Xue (sangue), direcionando-os para todo o corpo (YAMAMURA, 2004).

A acupuntura pode ser utilizada juntamente com o laser de baixa intensidade de energia, também conhecido como Laser Terapêutico, este por sua vez permite a estimulação dos acupontos e assim como a acupuntura é eficaz no tratamento de diversas patologias. Entre as mais diversas vantagens encontradas no laser acupunturam destaca-se a curta duração na aplicação, ótima opção para pacientes sensíveis a dor, fobias com agulhas, em

pessoas que apresentam problemas de coagulação ou que fazem tratamento com anticoagulantes (ALMEIDA-LOPES, 2010).

Sendo assim, nosso trabalho teve como objetivo, comparar o efeito da acupuntura e do laser acupuntura nos pontos E36 (Zusanli) e EX3 (Yintang) no perfil lipídico e glicêmico basal de ratos, assim como a concentração de cortisona basal, uma vez que esses acupontos sabidamente atuam diminuindo o estresse e agitação bem como estimulam a imunidade, atuando no equilíbrio do metabolismo e auxiliando na restauração das funções do organismo. 


\section{Materiais e método}

Todos os procedimentos experimentais foram aprovados pelo Comissão de Ética no Uso de Animais - CEUA da Universidade Regional de Blumenau (FURB), conforme protocolo $\mathrm{n}^{\circ}$ 017/17. Nele utilizamos 48 ratos Wistar (Rattus norvegicus) machos adultos (160-200g), provenientes do Biotério Central da FURB, Santa Catarina. Os ratos foram mantidos em biotério $\left(22 \pm 2^{\circ} \mathrm{C}\right)$ com ciclo claro-escuro de 12 horas e livre acesso a água e ração (ad libitum).

Para melhor desempenho do nosso trabalho, dividimos os animais em dois grupos de 24 animais, com a aclimatação de 15 dias no biotério do bloco T do campus 1 da FURB. Após esse período, foram divididos em 3 grupos de 8 animais, sendo: Grupo controle (sem intervenção), grupo acupuntura e laser. Previamente a experimentação, o primeiro grupo de 24 animais continuou a alimentação normal, já o segundo grupo de 24 animais ficaram em jejum por 12 horas. Para realização dos experimentos, todos os animais foram aclimatados no laboratório de bioquímica por um período de $1 \mathrm{~h}$. De forma integral, os procedimentos foram conduzidos de acordo com as normas de cuidados com animais de laboratório e as diretrizes éticas para investigação em animais conscientes (YIN et al., 2008).

Para inserção das agulhas, os animais foram gentilmente contidos e as agulhas colocadas uma a uma nos pontos E36 e EX3 onde permaneceram por 30 minutos unilateralmente (UM et al., 2005). Durante este período os animais foram observados para garantir que todas as agulhas permanecessem em seu ponto, caso o animal as removesse, elas eram imediatamente recolocadas. Utilizou-se agulhas de 0,18 x 8,0mm (Dong Bang). O Laser utilizado foi o de baixa intensidade (TF Premier Plus da MMO) com o comprimento de onda de $808 \mathrm{~nm}$ na faixa do infravermelho (analgésico) e a energia adicionada é de $1 \mathrm{~J}(25 \mathrm{~J} / \mathrm{cm} 2$, $100 \mathrm{~mW}$ por 10/s). Os pontos de referência foram escolhidos a partir dos efeitos observados em humanos e mapeados em ratos (LI; ZHANG; XIE, 2004).

Para a coleta das amostras os animais foram sedados com uma mistura de cetamina e xilazina $(70 \mathrm{mg} \backslash \mathrm{Kg}$ e $10 \mathrm{mg} \backslash \mathrm{Kg}$ respectivamente). Posteriormente, a amostra de sangue foi coletada por punção cardíaca e transferida imediatamente para tubo com gel separador e centrifugadas a 3500 RPM por 10 minutos. Para as análises, utilizou-se kits Bioclin ${ }^{\circledR}$ comercial. Para a dosagem do perfil lipídico utilizou-se analisador semiautomático Bioplus e o cortisona por sua vez, foi mensurado através da técnica de quimioluminescência.

Os resultados foram analisados no programa Graph Pad Prism versão 6. Eles foram expressos como média \pm erro padrão da média (E.P.M.) e avaliados através da análise de 
variância (ANOVA) de uma via seguido pelo teste pos-hoc de Newman-Keuls. Valores de p menores que $0,05(\mathrm{p}<0,05)$ foram considerados estatisticamente significativos.

\section{Resultados}

Perfil lipídico e glicêmico de ratos tratados com acupuntura e laser acupuntura (sem jejum)

A Figura 1 representa o perfil lipídico e glicêmico dos animais que receberam previamente o tratamento com acupuntura ou laser e comparados com o controle e entre si. Na Figura 1A, observa-se um aumento de aproximadamente $32 \%$ na concentração sérica de colesterol total no grupo tratado com laser quando comparado ao grupo controle e acupuntura.

$\mathrm{Na}$ análise da concentração de HDL-c (Figura 1B), por sua vez, pode-se verificar um aumento também significativo e próximo a $78 \%$ na concentração de HDL-c no grupo tratado com o laser em relação ao grupo controle, fato este que não foi observado no grupo tratado com acupuntura. Já a concentração dos Triglicérides, (Figura 1C), não demonstrou qualquer alteração significativa quando comparado laser acupuntura com acupuntura, ou cada um deles em comparação com o grupo controle.

Finalmente, em relação ao perfil glicêmico, observa-se que houve um aumento de aproximadamente $18 \%$ naquele grupo tratado com laser quando comparado aos grupos controle e acupuntura (Figura 1D).

Figura 1- Perfil lipídico e glicêmico (sem jejum). Colesterol Total (A), HDL-C (B), Triglicerídeos (C) e Glicose (D) média de 7- 8 animais. As barras verticais indicam o \pm EPM (erro padrão da média). $* \mathrm{P}<0,05$, ***P $<0,001$ comparado ao grupo controle; \#\# $\mathrm{P}<$ 0,01 comparado ao grupo da acupuntura. ANOVA de uma via seguida do teste de Newman Keuls.

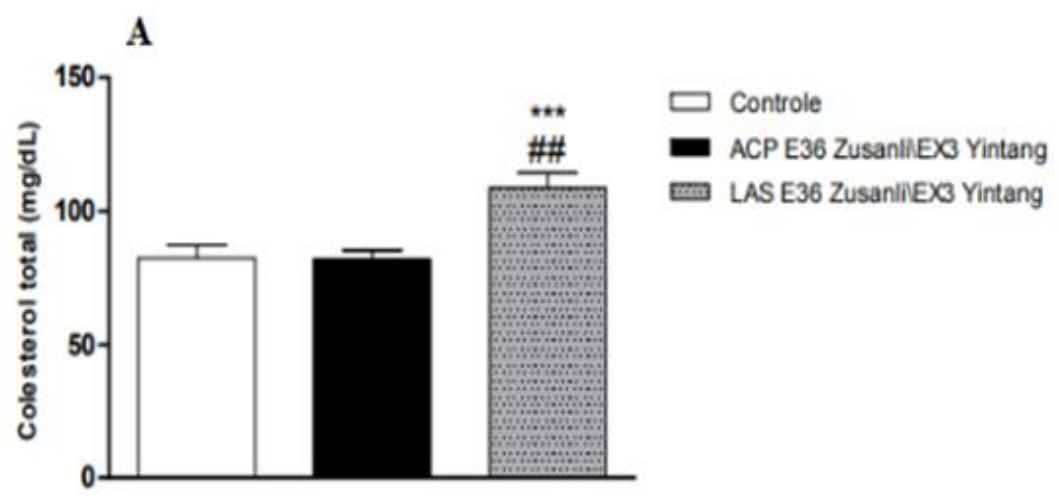



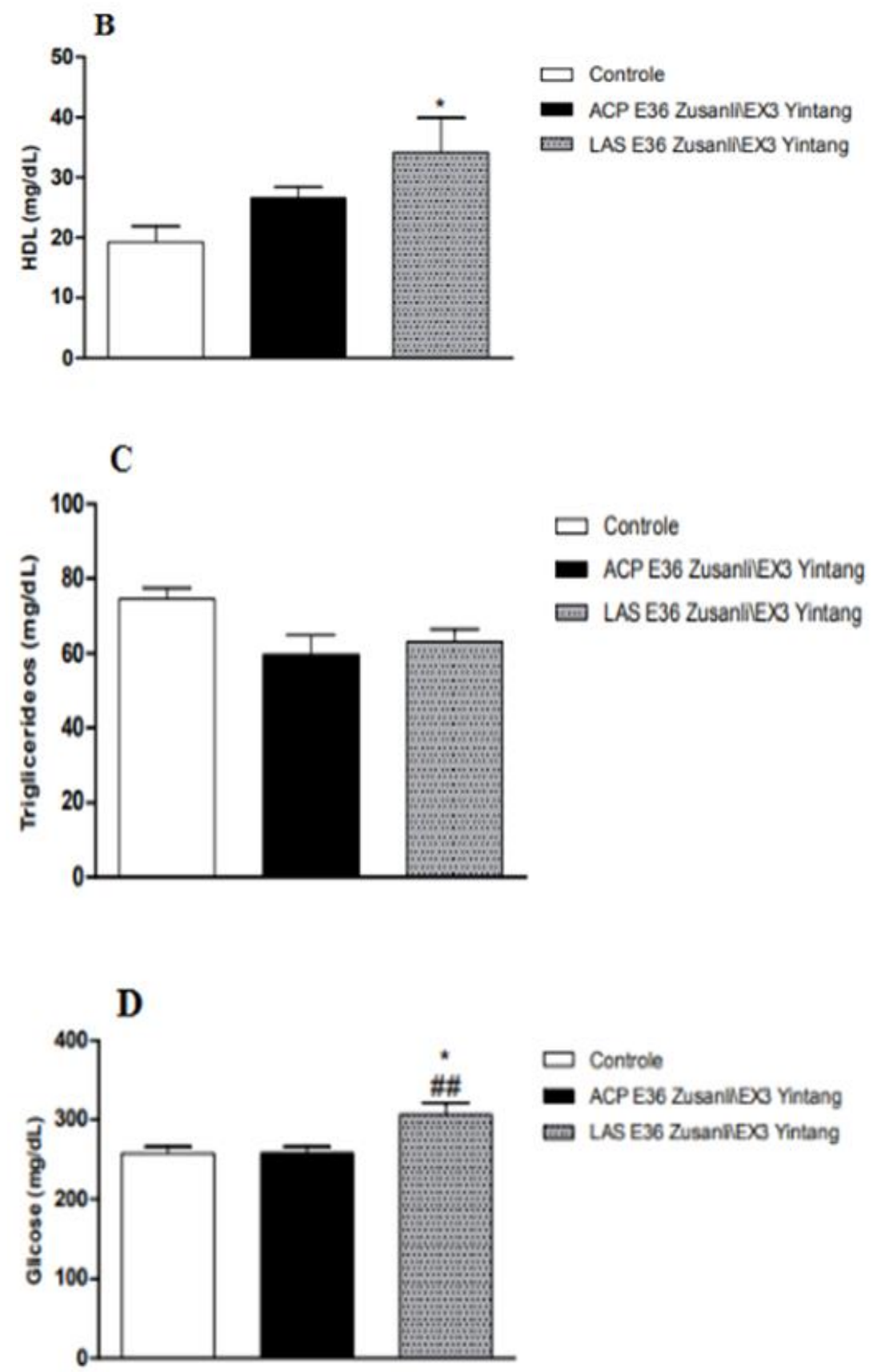

Perfil lipídico e glicêmico de ratos tratados com acupuntura e laser acupuntura (com jejum)

A Figura 2 representa o perfil lipídico e glicêmico dos animais que receberam previamente o tratamento com acupuntura ou laser e comparados com o controle e entre si. Na Figura 2A, verifica-se um aumento de aproximadamente $38 \%$ na concentração sérica de colesterol total no grupo tratado com laser quando comparado ao grupo controle, no entanto, entre os grupos laser e acupuntura não houve qualquer alteração. 
$\mathrm{Na}$ análise da concentração de HDL-c (Figura 2B), por sua vez, pode-se constatar um aumento também significativo e próximo a 39\% na concentração de HDL-c no grupo tratado com o laser em relação ao grupo controle, fato este que não foi observado no grupo tratado com acupuntura. Todavia, a concentração dos Triglicérides, (Figura 2C), não demonstrou qualquer alteração quando comparados os dois tratamentos com o grupo controle e entre si.

$\mathrm{Na}$ investigação da concentração do LDL-c, (Figura 2D), não se notou também qualquer alteração quando comparados os dois tratamentos com o grupo controle e entre si. Por fim, no que se refere às concentrações séricas do perfil glicêmico, (Figura 2E), novamente observa-se ausência de alterações significativas ditas nas duas amostras acima.

Figura 2- Perfil lipídico e glicêmico (com jejum). Colesterol Total (A), HDL-c (B), Triglicerídeos (C), LDL-c (D) e Glicose (E). Cada grupo representa a média de 7- 8 animais. As barras verticais indicam o $\pm \mathrm{EPM}$ (erro padrão da média). ${ }^{*} \mathrm{P}<0,05, * * \mathrm{P}<0,01$ comparado ao grupo controle; \#\# $\mathrm{P}<0,01$ comparado ao grupo da acupuntura. ANOVA de uma via seguida do teste de Newman Keuls

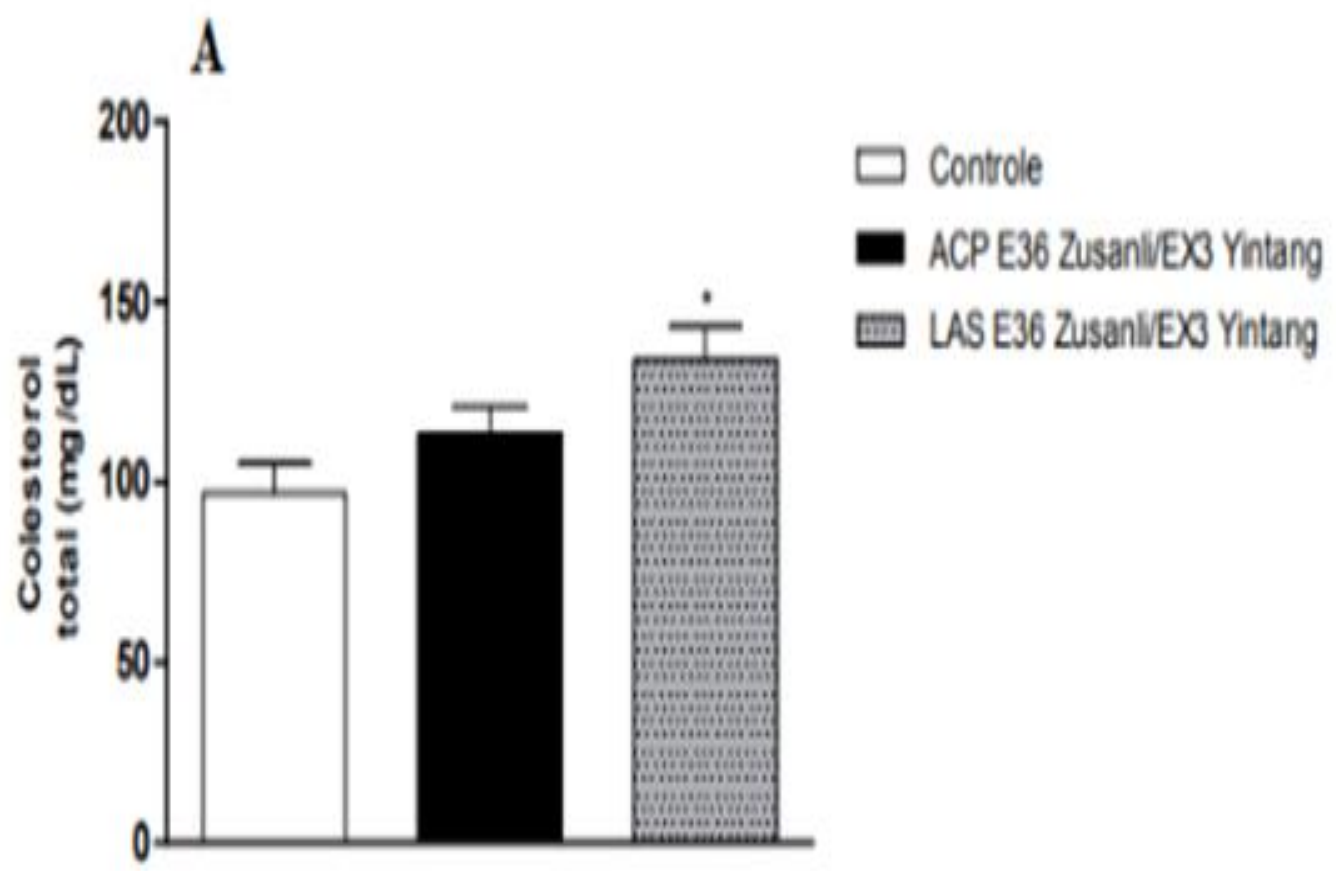




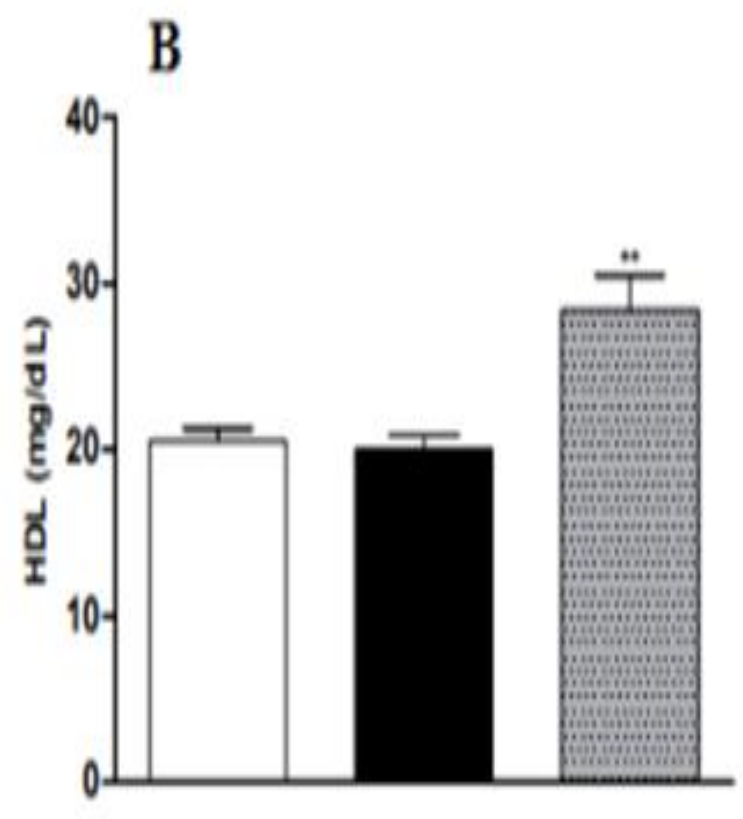

$\square$ Controle

- ACP E36 ZusanliEXO Yintang

$\square$ LAS E36 ZusaniEXX Yintang

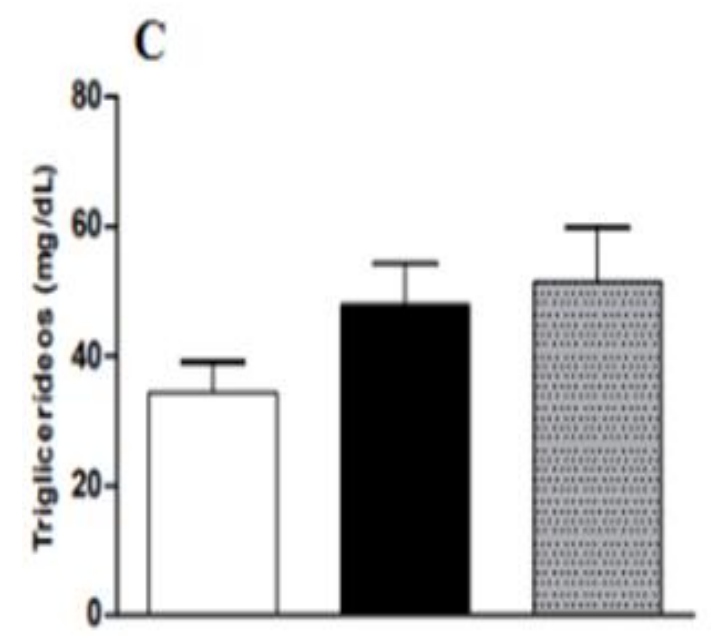

$\square$ Controle

- ACP E36 ZusaniliEX Yintang

LAS E36 ZusanVEX3 Yintang

D

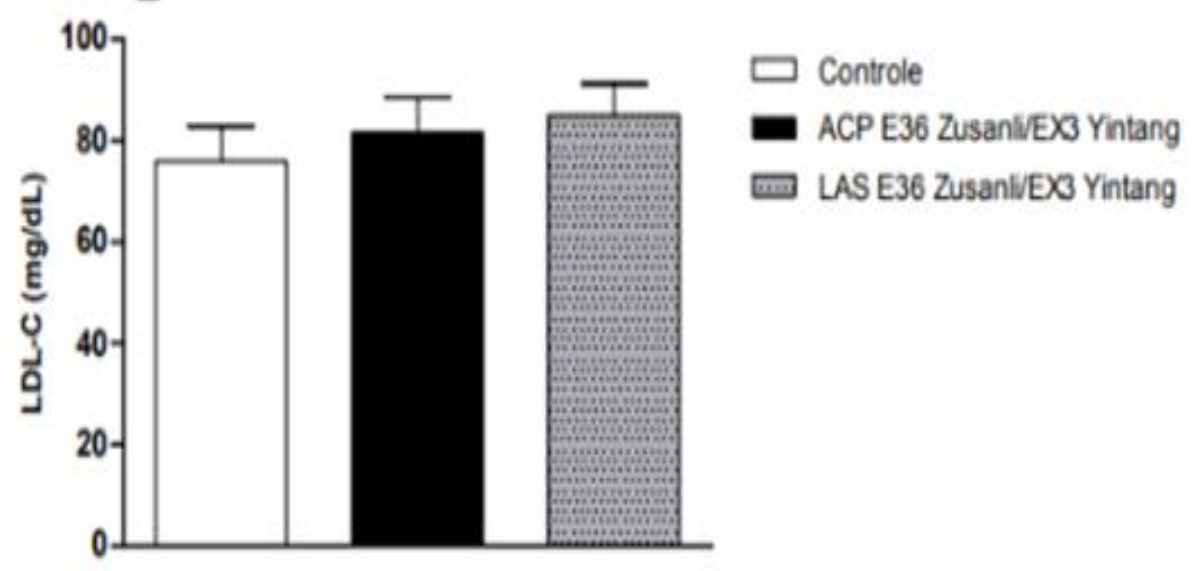




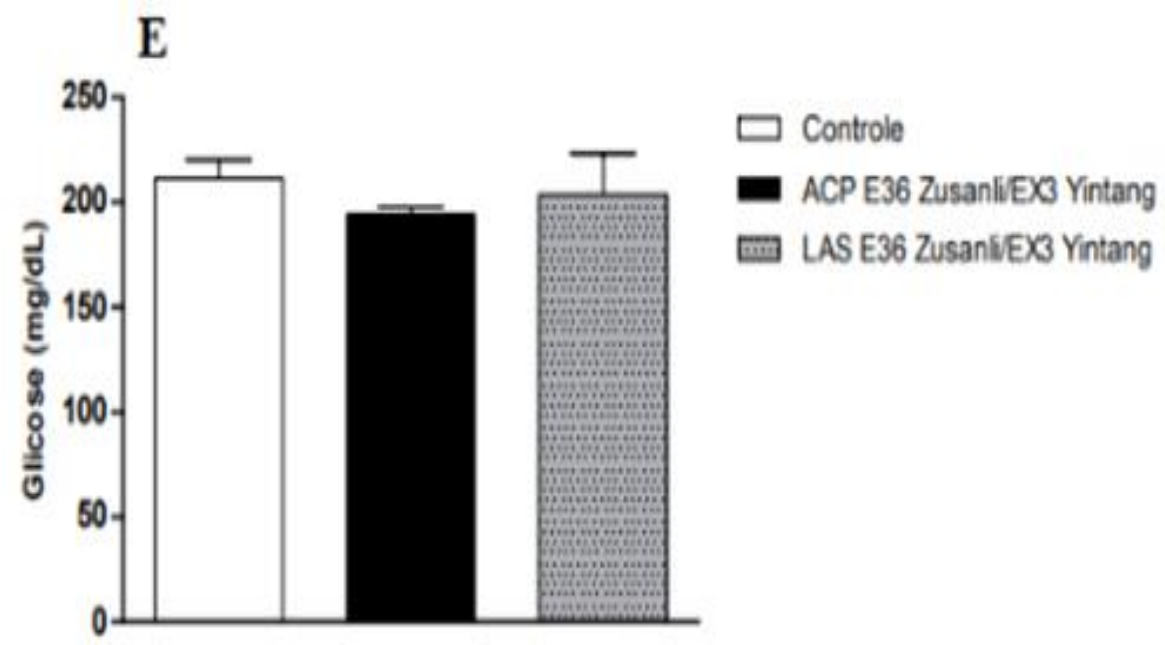

\section{Concentração do Cortisona em ratos tratados com acupuntura e laser acupuntura (com jejum)}

A Figura 3 representa a concentração sérica da cortisona em animais que receberam previamente o tratamento com acupuntura ou laser e comparados com o controle e entre si. Observando-se uma diminuição de aproximadamente $30 \%$ na concentração sérica no grupo tratado com laser quando comparado ao grupo controle, mas sem alteração quando observado no grupo tratado com acupuntura.

Figura 3- Concentração sérica de Cortisol (com jejum). Cada grupo representa a média de 7- 8 animais e as barras verticais indicam o \pm EPM (erro padrão da média). $* P<0,05$ comparado ao grupo controle. ANOVA de uma via seguida do teste de Newman Keuls.

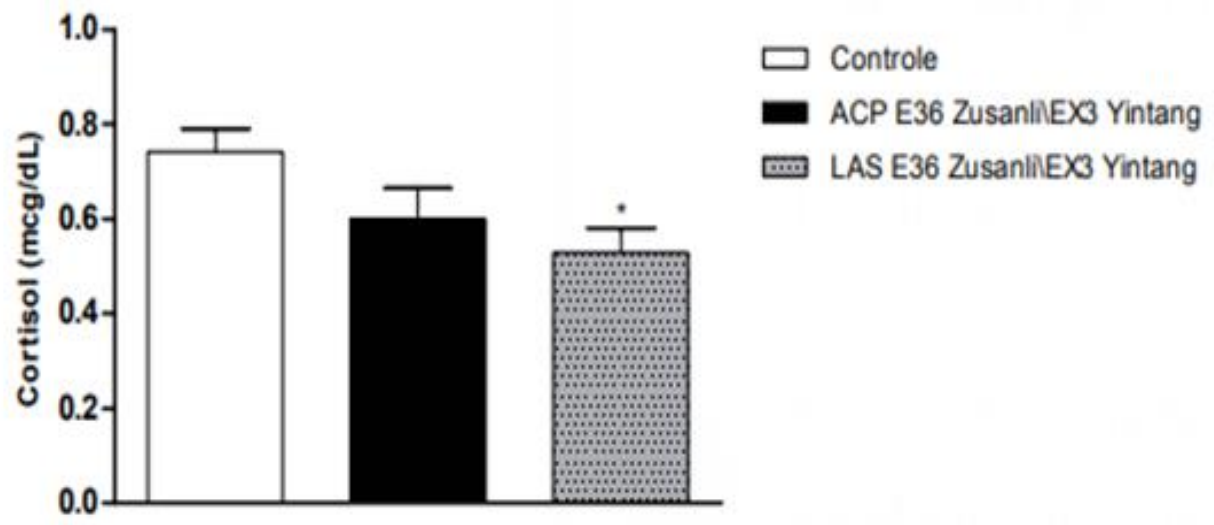




\section{Discussão}

Obteve-se no grupo sem jejum aumentos significativos do grupo laser acupuntura quando comparado com o grupo controle e acupuntura em alguns parâmetros, tais como colesterol total $32 \%$ e glicose $18 \%$, e no parâmetro de HDL-c teve um aumento significativo de $78 \%$ do grupo laser acupuntura comparado apenas ao grupo controle; quando avaliado o grupo com jejum, observa-se que a laser acupuntura em relação ao grupo controle teve um aumento significativo do colesterol total com 38\% e HDL-c com $39 \%$ e uma redução significativa da concentração do cortisona de aproximadamente $30 \%$.

Os mecanismos de ação da acupuntura não estão completamente estabelecidos, entretanto, sugere-se que a inserção e a manipulação das agulhas atuem como estímulo gerador de uma espécie de sinalização ao longo do tecido conjuntivo, disparando reações biomecânicas e bioquímicas relacionadas ao processo de cura (POVOLNY, 2008).

Ademais, sabe-se que os acupontos são extremamente reativos as correntes elétricas, e estes foram empiricamente determinados no transcorrer de milhares de anos de prática médica, e definidos como uma região da pele em que é grande a concentração de terminações nervosas sensoriais, tendo esta região relação íntima com nervos, vasos sanguíneos, tendões, periósteos e cápsulas articulares (ALBUQUERQUE,2018).

A acupuntura facilita ainda a atividade do sistema neuroendócrino e suas funções de regulação homeostáticas. Além disso, ela parece afetar a pressão sanguínea, motilidade gastrintestinal e o tempo de cicatrização de feridas (ANGELI; JOAQUIM; LUNA, 2007).

Foi analisado por meio da medicina esportiva equina, que a utilização da acupuntura produziu respostas neuroendócrinas como a diminuição na concentração plasmática de cortisol no homem e cortisona no cavalo, mas sem mencionar quais acupontos foram estimulados (ANGELI; JOAQUIM; LUNA, 2007). Nosso trabalho, por sua vez demonstra que o tratamento prévio dos animais com a acupuntura nos pontos E36\EX3 não foi capaz de promover uma significativa redução na concentração de cortisona.

Por outro lado, com o emprego da laserterapia, observamos alterações significativas havendo redução das concentrações plasmáticas de cortisona passados 30 min da estimulação dos acupontos E36lEX3. Diante disso, o laser é uma prática importante por permitir a estimulação de pontos que pode ser usada no tratamento das mais diversas patologias (WHITTAKER, 2004). 
Assim sendo, nossa hipótese é que o estresse, observado indiretamente pela concentração basal de cortisona, possa ter um efeito maléfico sobre o metabolismo, o crescimento, o processo de regeneração tecidual e a resposta imunológica.

Nesta mesma linha de pensamento, foi realizado um estudo avaliando a obesidade em animais, e constatou que a mesma se dá pelo menos em parte devido a um aumento da secreção de glicocorticoides. Em humanos, a obesidade parece ser seguida de vários sinais de disfunções hipotalâmicas semelhante ao observado em roedores, porém, em menor grau (MATOS; MOREIRA; GUEDES, 2003).

Sabe-se ainda, que a obesidade tem como característica uma produção aumentada de cortisona, combinada a uma elevada taxa de turnover, a qual resulta em uma cortisona circulante normal e, frequentemente, em concentrações mais baixos pela manhã (MATOS; MOREIRA; GUEDES, 2003).

Sugere-se ainda que a diminuição de substâncias como bradicinina, histamina e acetilcolina e a redução de citocinas e da enzima $\mathrm{COX} 2$, induzidas por parte da radiação à laser, proporcionam às células e tecidos, uma energia que intensifica em todos os níveis, o crescimento fisiológico normalizando as deficiências e equilibrando as concentrações de diversos biomarcadores (LINS et al., 2010).

Além disso, demonstrou-se que em situações de secreção elevada de cortisona, por meio da ativação do Sistema Nervoso Simpático (SNS), ocorrem alterações somáticas dentre as quais a mobilização de ácidos graxos livres (AGL) do tecido adiposo, exercendo efeitos como o desenvolvimento da resistência insulínica nos músculos e no fígado (MATOS; MOREIRA; GUEDES, 2003).

Em relação ao perfil lipídico, ainda não há meios para esclarecer totalmente alguns dos achados no presente estudo, muito embora já tenham apontado que em mulheres o tratamento com acupuntura sistêmica e auricular, podem ser utilizadas de forma segura e eficaz para tratar obesidade visto os fatores internos como as emoções desenvolvem 0 desequilíbrio energético o qual se manifestam nos órgãos ou vísceras (VIANA; MEJIA, 2008).

Por conseguinte, também foi avaliado as concentrações de Colesterol Total, HDL-c, Triglicérides e LDL-c. Sendo utilizado os seguintes pontos: E25, E36, PB6, P6 e E40, pontos referentes ao Estômago, Baço-Pâncreas e Pulmão, estes acupontos tem como função eliminar o alimento retido no Estômago, fazer o Qi circular pelo organismo fazendo os líquidos fluírem, eliminando a retenção de líquido que causa o inchaço abdominal e nos membros inferiores, principalmente. $\mathrm{Na}$ orelha os pontos utilizados por eles foram: Boca, Esôfago, 
Estômago, ShenMen, Endócrino e Pulmão, alternando as orelhas a cada sessão. Concluindose a correspondência entre a perda de peso e a diminuição das concentrações de colesterol totais e de triglicérides devido à utilização da prática integrativa (VIANA; MEJIA, 2008).

Em referência ao nosso estudo, diante dos resultados dos dois grupos, não houve diferença significativa no perfil lipídico entre os grupos que estiverem com jejum e o grupo sem jejum. Este resultado vai ao encontro com o que é preconizado pela "Normatização da Determinação Laboratorial do Perfil Lipídico" publicado no final de 2016 que tem como objetivo dispensar a necessidade de jejum de 12 horas para avaliação laboratorial do perfil lipídico. Sendo este Consenso desenvolvido por cinco sociedades médicas brasileiras: Sociedade Brasileira de Endocrinologia e Metabologia, Sociedade Brasileira de Diabetes, Sociedade Brasileira de Análises Clínicas, Sociedade Brasileira de Patologia Clínica e Medicina Laboratorial e Sociedade Brasileira de Cardiologia (SBPC, 2006).

Estes resultados confirmam estudos no qual apontam que ao fim de 12 a $24 \mathrm{~h}$ de jejum total, existe uma diminuição de, pelo menos, $20 \%$ na concentração de glicose no sangue (SERRANO, 2018).

Estudos anteriores mostram efeitos empíricos e clínicos da acupuntura auricular no tratamento da Diabetes Mellitus tipo 2 (DM2), mas ainda não há relatos de análises dos nossos acupontos E36 e EX3 com a glicemia. Da acupuntura auricular, perceberam que seus êxitos estiveram relacionados à ativação da enzima glicose-6-fosfato, com o crescimento da produção de insulina pelo pâncreas e aumento no número de receptores para insulina (ASSIS, 2018).

\section{Conclusão}

Concluiu-se que na avaliação do efeito entre a acupuntura e o laser acupuntura nos pontos E36 (Zusanli) e EX3 (Yintang) para verificar o perfil lipídico, glicemia sérica e a concentração de cortisona, o laser acupuntura teve uma resposta mais robusta em relação à acupuntura e o controle.

Por outro lado, nesse estudo trabalhamos com associação de acupontos, E36 e EX3, em temas como tratamento do perfil lipídico, glicêmico e da cortisona na acupuntura e laser acupuntura que são totalmente inovadores, necessitando, desta forma, mais detalhamento e pesquisas para aprimorar a discussão do conteúdo apresentado.

Acreditamos que a ciência é uma construção, em que sempre há de se colocar ou retirar um tijolo, onde não existe julgamentos certos ou errados, mas uma contínua 
movimentação do conhecimento, o qual é sempre necessário para busca do aperfeiçoamento dos temas e da melhoria para saúde.

Dessa maneira, o nosso trabalho tem o propósito de estimular ainda mais os estudos sobre terapias complementares, especialmente a acupuntura e laser acupuntura, pois são métodos complementares à saúde e profiláticas, reequilibrando funcionalmente o organismo. Assim, como o próprio nome exemplifica, elas são para complementar a prática médica além das farmacoterapias típicas e buscas diminuir os efeitos colaterais dessas.

\section{Referências}

ALBUQUERQUE, A.P. O efeito da microcorrente sobre o acuponto zusanli nos modelos de dor incisional, inflamatória e neuropática em ratos wistar. Alfenas, 2018. Dissertação (Mestrado em Biociências Aplicada à Saúde), Universidade Federal de Alfenas.

ALMEIDA-LOPES, L. Laserpuntura: Bases Científicas e Aplicações - Revisão Bibliográfica. São Carlos, 2010. Monografia (Especialização em Medicina Tradicional Chinesa), Escola Spaço Alternativo.

ANGELI, A.L.; JOAQUIM, J.G.F.; LUNA, S.P.L. ACUPUNTURA APLICADA À MEDICINA ESPORTIVA EQÜINA. Revista Acadêmica: Ciência Animal, v. 5, n. 3, p. 325333, 2007.

ASSIS, B.B. O efeito da acupuntura auricular sobre o risco do pé diabético: ensaio clínico controlado e mascarado. Alfenas, 2018. Dissertação (Mestrado em Enfermagem), Universidade Federal de Alfenas.

BRASIL. Ministério da Saúde. Secretaria de Atenção à Saúde. Departamento de Atenção Básica. Política Nacional de Práticas Integrativas e Complementares no SUS PNPICSUS. Ministério da Saúde, Secretaria de Atenção à Saúde, Departamento de Atenção Básica. - Brasília: Ministério da Saúde, 2006.

COSTA, P.H. A acupuntura no sistema único de saúde. Revista Brasileira de Ciências em Saúde-Brazilian Journal of Health Sciences, v. 1, n. 1, p. 58-63, 2017.

LI, A.H.; ZHANG, J.M.; XIE, Y.K. Human acupuncture points mapped in rats are associated with excitable muscle/skin-nerve complexes with enriched nerve endings. Brain Res. v.1012, n.1-2, p.154-159, 2004.

LINS, R. D. A. U. D., E. M.; LUCENA, K. C. R.; CATÃO, M. H. C. V.; GRANVILLEGARCIA, A. F.; NETO, L. G. C. Efeitos bioestimulantes do laser de baixa potência no processo de reparo. An Bras Dermatol, v.85, n.6, p.849-55, 2010.

MATOS, A.F.G.; MOREIRA, R.O.; GUEDES, E.P. Aspectos neuroendócrinos da síndrome metabólica. Arq Bras Endocrinol Metabol, v. 47, n. 4, p. 410-420, 2003.

YAMAMURA, Y. Acupuntura tradicional. A arte de inserir. São Paulo: Ed. Roca, 2004.

POVOLNY, B. Acupuncture and traditional Chinese medicine: an overview. Techniques in Regional Anesthesia and Pain Management, v. 12, n. 2, p. 109-110, 2008.

SBPC - Sociedade Brasileira de Patologia Clínica. Consenso Brasileiro para a Normatização da Determinação Laboratorial do Perfil Lipídico: Flexibilização do Jejum para a avaliação do 
Perfil Lipídico. Rio de Janeiro, 2016. Acesso em: 02/03/2019. Disponivel em: http://www.sbpc.org.br/upload/conteudo/consenso_jejum_dez2016_final.pdf

SERRANO, M.S. O jejum intermitente como dieta alternativa no tratamento da Diabetes Mellitus tipo 2. Porto, 2018. Dissertação (Mestrado em Medicina), Universidade do Porto.

UM, S.W.; KIM, M.S.; LIM, J.H.; KIM, S.Y.; SEO, K.M.; NAM, T.C. Thermografic evaluation for the efficacy of acupuncture on induced chronic arthritis in the dog. J. Vet. Med. Sci. v. 67, n.12, p.1283-1284, 2005.

VIANA, M.J.; MEJIA, D.P.M. Acupuntura como recurso no tratamento da obesidade. Pósgraduação em Acupuntura-Faculdade Ávila, 2008.

WHITTAKER, P. Laser acupuncture: past, present, and future. Lasers in Medical Science, v. 19, p. 69-80, 2004.

YIN, C.S.A; HYEOK-SANG, J.B; HI-JOON, P.C.; YOUSANG, B.D.; MOONHYUN, Y.E.; CHI-BONG, C.F.; HYEONG, G.K. A proposed transpositional acupoint system in a mouse and rat model. Research in Veterinary Science. v.84, p.159-165, 2008.

Recebido em 15/09/2019. Aprovado em 30/11/2019. 